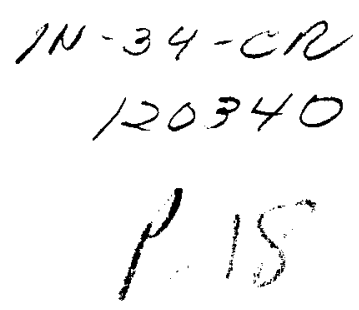

SEMI-ANNUAL STATUS REPORT

June 1897 - January 1988

for

NASA Grant NAG-1-778

(AASA-CR-182417) A CCEFUTATI(AALIY

EFFICIEAT MCDELIIAG OF LALINAE SEEAEATICN

N88-15949

EUBBLES Semiannual Status kefCrt, Jun. 1987

- Jan. 1588 (Pensyluaria state Univ.)

$18 \mathrm{p}$

CSCL 20D G3/34 0120340

\title{
A COMPUTATIONALLY EFFICIENT MODELLING \\ OF LAMINAR SEPARATION BUBBLES
}

\author{
Mark D. Maughmer \\ Principal Investigator \\ Department of Aerospace Engineering \\ The Pennsylvania State University \\ University Park, PA 16802
}

February, 1988 


\section{SEMI-ANNUAL STATUS REPORT}

June 1897 - January 1988

for

NASA Grant NAG-1-778*

The goal of this research is to accurately predict the characteristics of the laminar separation bubble and its effects on airfoil performance. To this end, a model of the bubble is under development and will be incorporated in the analysis section of the Eppler and Somers program. ${ }^{1}$ As a first step in this direction, an existing bubble model has been inserted into the program. This has the dual purpose of gaining insight into the correlation between existing theory and bubble prediction trends, as well as becoming familiar with the analytical and numerical details of Eppler's boundary-layer analysis method.

The selection of the first bubble model to be used in the Eppler and Somers program was based on several considerations. In the first place, it was decided to address the problem of the short bubble before attempting the prediction of the long bubble. The short bubble entails, by definition, a much weaker interaction between the viscous and inviscid regions of the flow field than the long bubble. This, and the greater amount of experimental data available for the short bubble, renders the development of a short-bubble model more tractable both analytically and numerically. In the second place, an integral boundary-layer method is believed more desirable than a finite difference approach. While these two methods achieve similar prediction accuracy, finite-difference methods tend to involve significantly longer computer run times than the integral methods. Since the Eppler and Somers program is primarily an airfoil design program, computational efficiency of the bubble model is of primary importance. Finally, as the boundary-layer analysis in the Eppler and Somers program employs the momentum and kinetic energy integral equations, a short-bubble model compatible with these equations is most preferable.

* NASA Technical Monitor for this Grant is Mr. Dan M. Somers, NASA Langley Research Center, Hampton, VA 23665 
The short-bubble model of Horton ${ }^{2}$ meets with all the above considerations and was therefore chosen for incorporation into the program. In addition, some modifications based on experimental data more recent than that available to Horton have been included. Specifically, the length of the laminar length is based on a curve fit, calculated by O'Meara and Mueller ${ }^{3}$ of Reynolds number based on the laminar length as a function of Reynolds number based on the momentum thickness at separation. Values suggested by Roberts ${ }^{4}$ for the mean dissipation coefficient in the turbulent separated shear layer and for the reattachment parameter have been used. The bubble is matched to Eppler's laminar separation criterion and turbulent boundary-layer method. In spite of these improved correlations and empirical constants, this model remains quite crude. As will be shown below, however, this crudeness has helped to isolate those areas in the bubble region and in the integral boundary-layer method that will need further study before a faithful reproduction of the pertinent physical processes can be achieved.

\section{Analytical and Numerical Details}

The modified Horton bubble model which has been incorporated into the Eppler and Somers program is triggered when Eppler's laminar separation criterion is met; that is, when

$$
\left(H_{32}\right)_{S}=1.515095
$$

As discussed by Horton, in the laminar portion of the bubble the streamwise pressure gradient is assumed to equal zero. Thus,

$$
U_{T}=U_{S}
$$

Further, the assumption of negligible skin friction leads, from the momentum integral equation, to

$$
\left(\delta_{2}\right)_{T}=\left(\delta_{2}\right)_{S}
$$

O'Meara and Mueller have correlated the length of the laminar shear layer to the momentum thickness at separation, based on seven different sets of data including their own, and 
found

$$
R e_{\ell_{1}}=155 R e_{\left(\delta_{2}\right)}
$$

or,

$$
\frac{\ell_{1}}{c}=155 \frac{\left(\delta_{2}\right)_{s}}{c}
$$

This correlation matches well more recent data taken by Brendel and Mueller, ${ }^{5}$ and was therefore used in place of Horton's original transition criterion,

$$
R e_{\ell_{1}}=40000
$$

In order to proceed from the transition point to the turbulent reattachment point, a number of additional assumptions are made. As discussed in Ref. 2, it appears from experimental data that

$$
\left[\frac{d H_{32}}{d s}\right]_{R}=0
$$

Based on this result, and on the vanishing of skin friction at a point of reattachment, Horton was able to reduce Truckenbrodt's shape parameter equation to

$$
\left[\frac{\delta_{2}}{U} \frac{d U}{d s}\right]_{R}=-\left[\frac{C_{D}}{H_{32}\left(H_{12}-1\right)}\right]_{R}=\Lambda_{R}
$$

The assumption of a universal reattachment velocity profile and a constant $C_{D}$ in the turbulent part of the bubble leads to a constant value for $\Lambda_{R}$. Roberts suggests an experimentally determined mean value of the dissipation coefficient,

$$
C_{D}=0.035
$$

Using the generally accepted values of

$$
\left(H_{32}\right)_{R}=1.51
$$

and

$$
\left(H_{12}\right)_{R}=3.5
$$


Eq. (7) gives

$$
\Lambda_{R}=-0.0093
$$

As discussed in Ref. 6, both Horton and Roberts attempted to measure $\Lambda_{R}$. The mean of their values was

$$
\Lambda_{R}=-0.0075
$$

In a later publication, ${ }^{4}$ however, Roberts explains that the best agreement with experimental data was obtained by using Horton's original theoretical value for the dissipation coefficient,

$$
C_{D}=0.022
$$

such that

$$
\Lambda_{R}=-.0059
$$

Clearly, there are a number of contradictions among these results. For the time being, the values in Eqs. (8) and (12) are used in the bubble model.

Assuming a constant $C_{D}$ and $H_{32}$, Eqs. (8) and (9), and a linear pressure recovery from transition to reattachment, the energy integral equation can be integrated to obtain the momentum thickness at reattachment:

$$
\left(\delta_{2}\right)_{R}=\left(\delta_{2}\right)_{T}\left(\frac{U_{T}}{U_{R}}\right)^{3}+\ell_{2}\left(\frac{C_{D}}{4 H_{32}}\right)\left(1+\frac{U_{T}}{U_{R}}\right)\left[1+\left(\frac{U_{T}}{U_{R}}\right)^{2}\right]
$$

Eliminating $\left(\delta_{2}\right)_{R}$ between Eqs. (7) and (15),

$$
\ell_{2}=\frac{\left(\delta_{2}\right)_{T}\left(\frac{U_{T}}{U_{R}}\right)^{3}\left(1-\frac{U_{T}}{U_{R}}\right)}{\Lambda_{R}-\left(\frac{C_{D}}{4 H_{32}}\right)\left[1-\left(\frac{U_{T}}{U_{R}}\right)^{4}\right]}
$$

In order to implement this result numerically, $U_{R}$ is decreased in small increments from the value of $U_{T}$. At each step, $\ell_{2}$ is calculated and it is checked whether the segment joining $T$ to $R$ intersects the inviscid velocity distribution as shown in Fig. 1. The turbulent calculations start at $s_{R}$, with the size of the first step equal to $X B$. This is the distance from $s_{R}$ to the first airfoil coordinate after reattachment. The drag coefficient is then determined from the Squire-Young formula. 


\section{Preliminary Results and Discussion}

Given all the assumptions discussed above, the modified Horton model cannot be expected to perform well in its present form. In addition, most of the assumptions implemented are based on measurements made at high Reynolds numbers, and are not necessarily correct at low Reynolds numbers. A good example of the weakness of this model is given by an analysis of the Eppler 387 airfoil, designed to operate at $R e=300,000$. The aerodynamic characteristics predicted using the original Eppler analysis and those from the program with the bubble model included are compared in Fig.2. Some points from the recent measurements of McGhee, et al. ${ }^{7}$ are also included. The original Eppler analysis slightly underpredicts the drag, possibly because it does not account for the drag due to the bubble. Inclusion of the modified Horton model, as detailed above, however, likewise does not reproduce the experimental data. In fact, at the lower lift coefficients the drag predicted with the bubble model is lower than that predicted without it. In the mid- $C_{\ell}$ range, the prediction using the bubble model exhibits a large region of turbulent separated flow. Near $\alpha=10^{\circ}$ from zero lift, the length of the turbulent separated region upstream of the trailing edge decreases and the lift increases again.

The low drag at low $C_{\ell}$ 's is believed to be a consequence of assuming no growth in momentum thickness from separation to transition. This assumption appears to be valid for $R e>1,000,000$ but, as shown by the data of Brendel and Mueller, not at low Reynolds numbers. The cause for the high drag and separated flow in the mid- $C_{\ell}$ range is not clear. While the bubble length does not change significantly from its length at the lower angles of attack, the actual upper surface pressure levels may be noticeably lower than the inviscid values, especially upstream of the bubble. In other words, using the value of $U_{S}$ from the

inviscid velocity distribution leads to a higher gradient across the bubble and the rest of the boundary layer than is present in reality; hence the deterioration in performance. As can be seen from the transition curves, the decreasing size of the separated flow length at the high $C_{\ell}$ 's is caused by the shift in bubble location from mid-chord to the leading edge.

In order to better observe the details of the boundary-layer solution which lead to 
the behavior just discussed, a new series of plots was generated. For each angle of attack of interest, the airfoil analyzed, the velocity distribution, the boundary-layer data, and the viscous analysis summary are all shown together on one page. This makes it possible to view all the relevant relationships simultaneously. Fig. 3a contains the results of the analysis at $\alpha=1.52^{\circ}$, the low end of the polar. The upper surface exhibits a fairly long mid-chord bubble, while the lower surface a short leading-edge bubble. The boundarylayer data is plotted in the form used by Eppler as $\log _{10}\left(R e_{\delta_{2}}\right)$ vs. $H_{32}$, as well as in the four plots of $H_{12}, \delta_{2}, H_{32}$, and $c_{f}$ vs. $s / c$. Moreover, the results from the original Eppler method and those from the method with the bubble model are plotted together to aid in the comparison. To help in the interpretation of the data, two modifications were made to the original Eppler and Somers program. First, the actual separation and reattachment points are plotted in both the $\log$ and the linear plots and second, in the log plot, all the points for which $H_{32}>1.62$ are plotted with $H_{32}=1.62$ rather than being skipped altogether.

At $\alpha=2.6^{\circ}$, Fig. $3 \mathrm{~b}$, the character of the bubble on the upper surface has not changed, while the flow on the lower is all laminar. At $\alpha=5^{0}$, Fig. 3c, it is clear that the model is not incrementing the momentum thickness correctly. In fact, the value at reattachment equals the value obtained by assuming transition at the laminar separation point. Incorporating an empirical correlation for the growth in $\delta_{2}$ in the laminar part of the bubble should improve the results. At $\alpha=8^{\circ}$, Fig. $3 \mathrm{~d}$, the bubble causes the flow to separate near the trailing edge. The higher drag coefficient is due to the separation as well as to an increase in $\delta_{2}$ across the bubble which is higher than that through the same length of turbulent boundary layer. The dependence of the increment in $\delta_{2}$ on angle of attack is related to the increasing steepness the inviscid pressure recovery as $\alpha$ increases, the length of the bubble remaining approximately constant. At $\alpha=10.48^{\circ}$, Fig. $3 e$, this prediction trend is most noticeable. The fact that now the drag is even higher than the experimental values might be explained by the inability of this model to account for viscous/inviscid interaction. In fact, as long as the separated shear layer reattaches, 
any adverse pressure gradient will drive the growth in boundary-layer properties across the bubble with no chance for them to "backfire" and mitigate the too severe pressure gradient. As shown in Fig. 23c in Ref. 7, however, the inviscid pressure distribution, even at $\alpha=7.36^{\circ}$, has been modified. In particular, the rooftop, which is immediately upstream of the bubble, is lower, while the pressure at reattachment is closer to the inviscid value. On the other hand, from the observed data, a long bubble does not appear to be present, in the sense that the inviscid velocity distribution is not modified drastically. Therefore, in order to correctly capture the bubble drag, it may be sufficient to decrease the value of the velocity at separation obtained from the inviscid pressure distribution by means of an empirical factor. Such factor could be correlated to Reynolds number and angle of attack. This approach assumes that the history of the laminar boundary layer from stagnation to separation is not of primary importance. If such an assumption were to prove faulty, it might then be left as the only alternative to perform a displacement thickness iteration assuming transition at laminar separation, and then to apply a short-bubble model to the resulting "viscous" pressure distribution. At $\alpha=10.49^{\circ}$, Fig. 3f, the laminar boundary layer separates upstream, near the leading edge, and a short bubble again forms.

A further aspect of this model that will need to be treated correctly is the match to Eppler's turbulent boundary-layer method at reattachment. Horton's model, and the reattachment parameter $\Lambda_{R}$, depend on the experimental values for $\left(H_{32}\right)_{R}=1.51$ and $\left(H_{12}\right)_{R}=3.5$. Eppler, however, uses a correlation for $H_{12}=H_{12}\left(H_{32}\right)$ that yields $H_{12}=$ 2.34 if $H_{32}=1.51$. This can be seen in all the plots described above. In other words, no allowance is made for that region, downstream of reattachment, in which the turbulent boundary layer is redeveloping and approaching a state for which Eppler's correlations are valid. The importance of an accurate modelling of this region is not yet known. 


\section{References}

1. Eppler, R., and Somers, D. M., "A Computer Program for the Design and Analysis of Low-Speed Airfoils," NASA TM-80210, 1980.

2. Horton, H. P., "A Semi-empirical Theory for the Growth and Bursting of Laminar Separation Bubbles," Aeronautical Research Council C. P. 1073, June 1967.

3. O'Meara, M. M., and Mueller, T. J., "Experimental Determination of the Laminar Separation Bubble Characteristics of an Airfoil at Low Reynolds Numbers," AIAA Paper 86-1065, May 1986.

4. Roberts, W. B., "Calculation of Laminar Separation Bubbles and Their Effects on Airfoil Performance," AIAA Paper 79-0285, January 1979.

5. Brendel, M., and Mueller, T. J., "Boundary Layer Measurements on an Airfoil at Low Reynolds Numbers," AIAA Paper 87-0495, January 1987.

6. Schmidt, G. S., "The Prediction of Transitional Separation Bubbles at Low Reynolds Numbers," Ph.D. Thesis, Department of Aerospace and Mechanical Engineering, University of Notre Dame, December 1986.

7. McGhee, R. J., Jones, G. S., and Jouty, R., "Performance Characteristics from WindTunnel Tests of a Low-Reynolds-Number Airfoil," AIAA Paper 88-0607, January 1988. 


\begin{tabular}{|c|c|}
\hline$c$ & $=$ Airfoil chord \\
\hline$c_{f}$ & $=$ Skin-friction coefficient \\
\hline$\ell_{1}$ & $=$ Laminar length of the bubble \\
\hline$\ell_{2}$ & $=$ Turbulent length of the bubble \\
\hline$s$ & $=$ Streamwise coordinate from the stagnation point \\
\hline$C_{d}$ & $=$ Sectional drag coefficient \\
\hline$C_{D}$ & $=$ Dissipation coefficient \\
\hline$C_{\ell}$ & $=$ Sectional lift coefficient \\
\hline$C_{m}$ & $=$ Sectional moment coefficient about $c / 4$ \\
\hline$H_{12}$ & $=$ Boundary layer shape factor, $\left(\delta_{1} / \delta_{2}\right)$ \\
\hline$H_{32}$ & $=$ Boundary layer shape factor, $\left(\delta_{3} / \delta_{2}\right)$ \\
\hline$R e_{\ell_{1}}$ & $=$ Transition Reynolds number, $\left(U_{S} \ell_{1} / \nu\right)$ \\
\hline$R e_{\delta_{2}}$ & $=$ Momentum thickness Reynolds number, $\left(U \delta_{2} / \nu\right)_{S}$ \\
\hline$U$ & $=$ Velocity at the edge of the boundary layer \\
\hline$U_{\infty}$ & $=$ Free sream velocity \\
\hline$X B$ & $=$ Distance from $s_{R}$ to first airfoil coordinate after $s_{R}$ \\
\hline$X U$ & $=$ Laminar fraction within one boundary layer step \\
\hline$\alpha$ & $=$ Angle of attack relative to the zero-lift line \\
\hline$\delta_{1}$ & $=$ Boundary layer displacement thickness \\
\hline$\delta_{2}$ & $=$ Boundary layer momentum thickness \\
\hline$\delta_{3}$ & $=$ Boundary layer kinetic energy thickness \\
\hline$\nu$ & $=$ Kinematic viscosity of air \\
\hline$\Lambda_{R}$ & $=$ Horton's reattachment parameter \\
\hline \multicolumn{2}{|c|}{ Subscripts: } \\
\hline$S$ & $=$ Laminar separation point \\
\hline$R$ & $=$ Turbulent reattachment point \\
\hline$T$ & $=$ Transition point \\
\hline
\end{tabular}




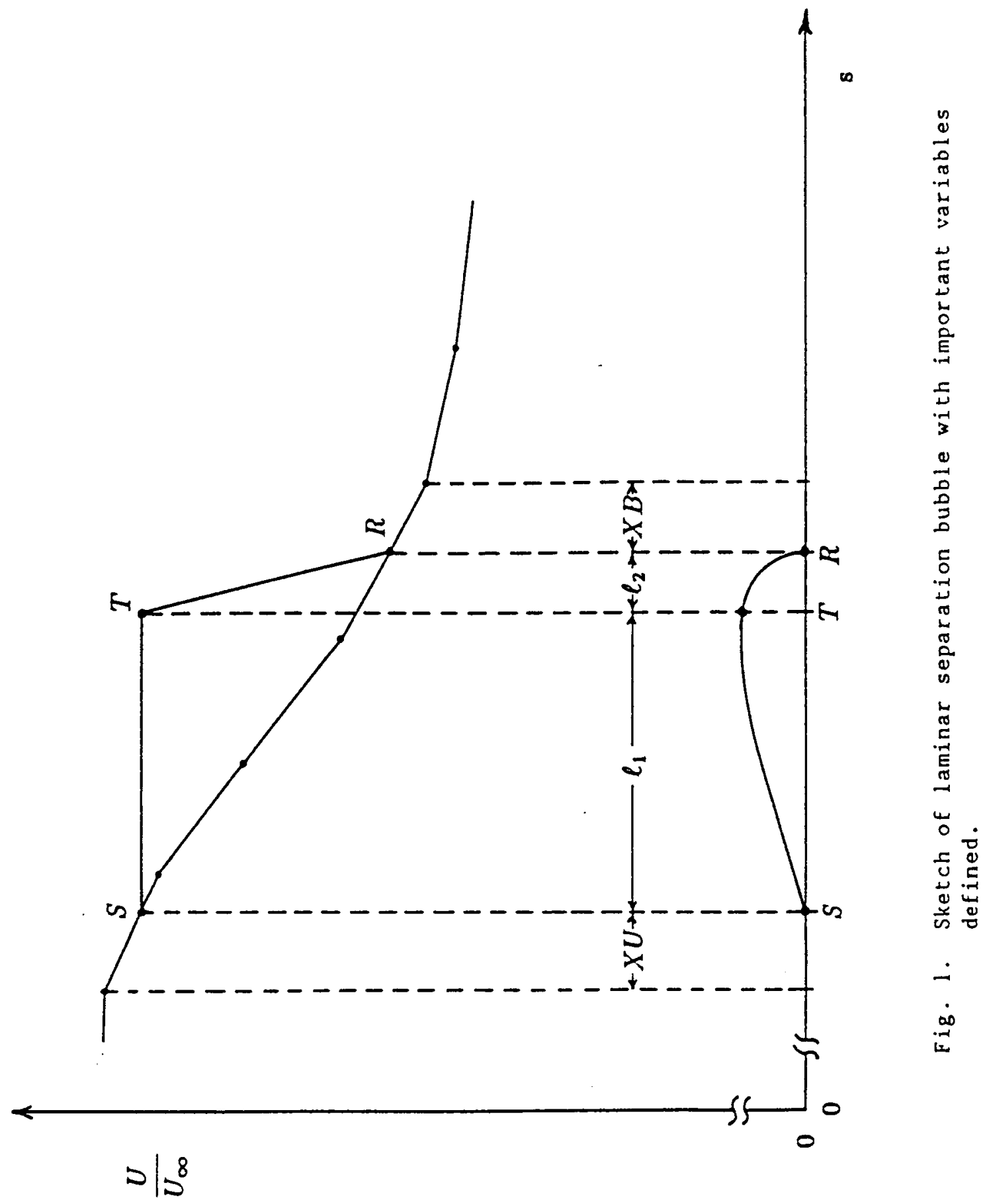




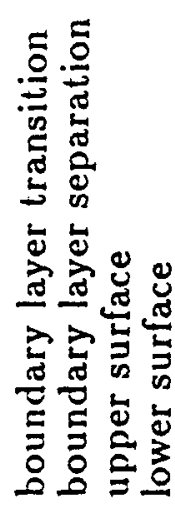

|| || || ||

نं

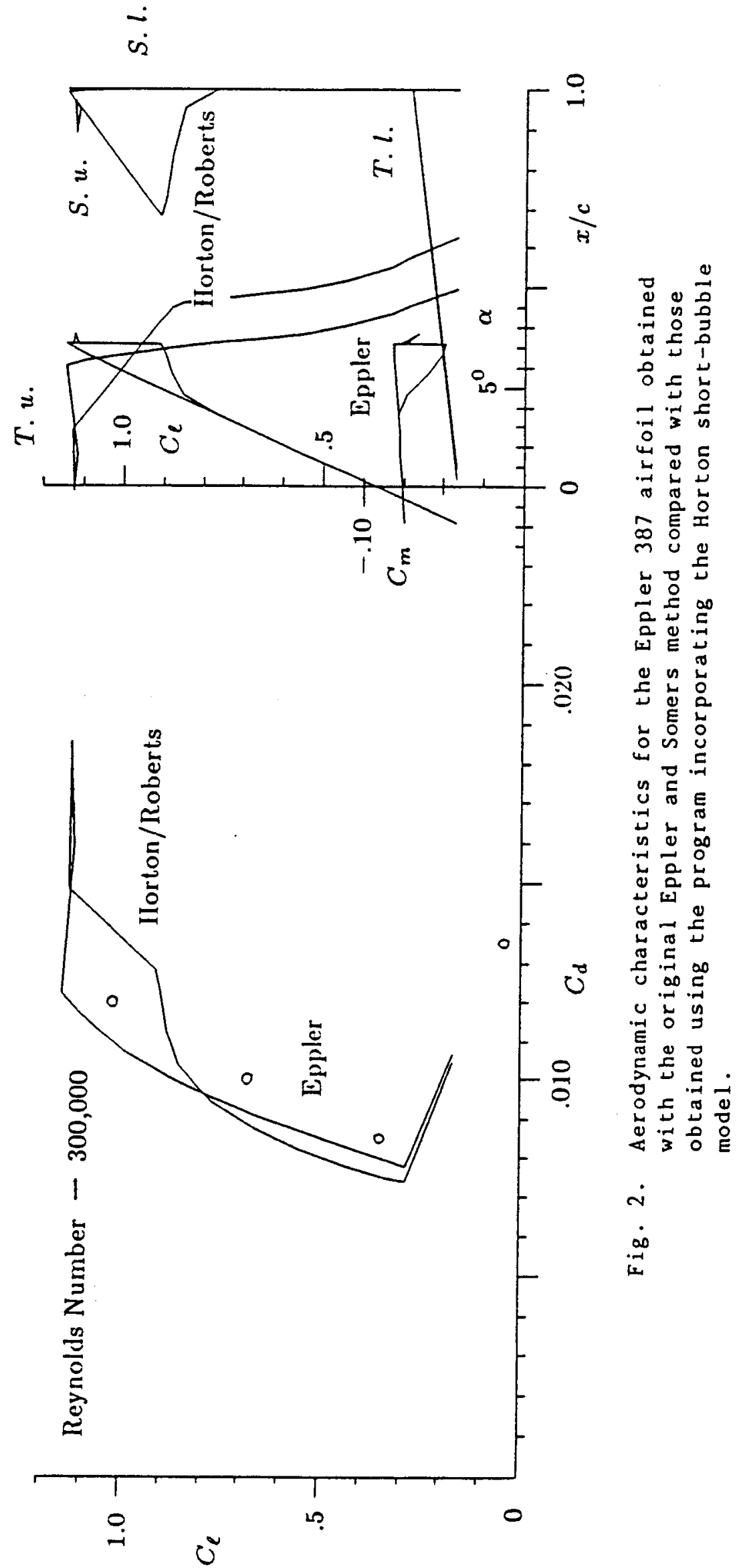




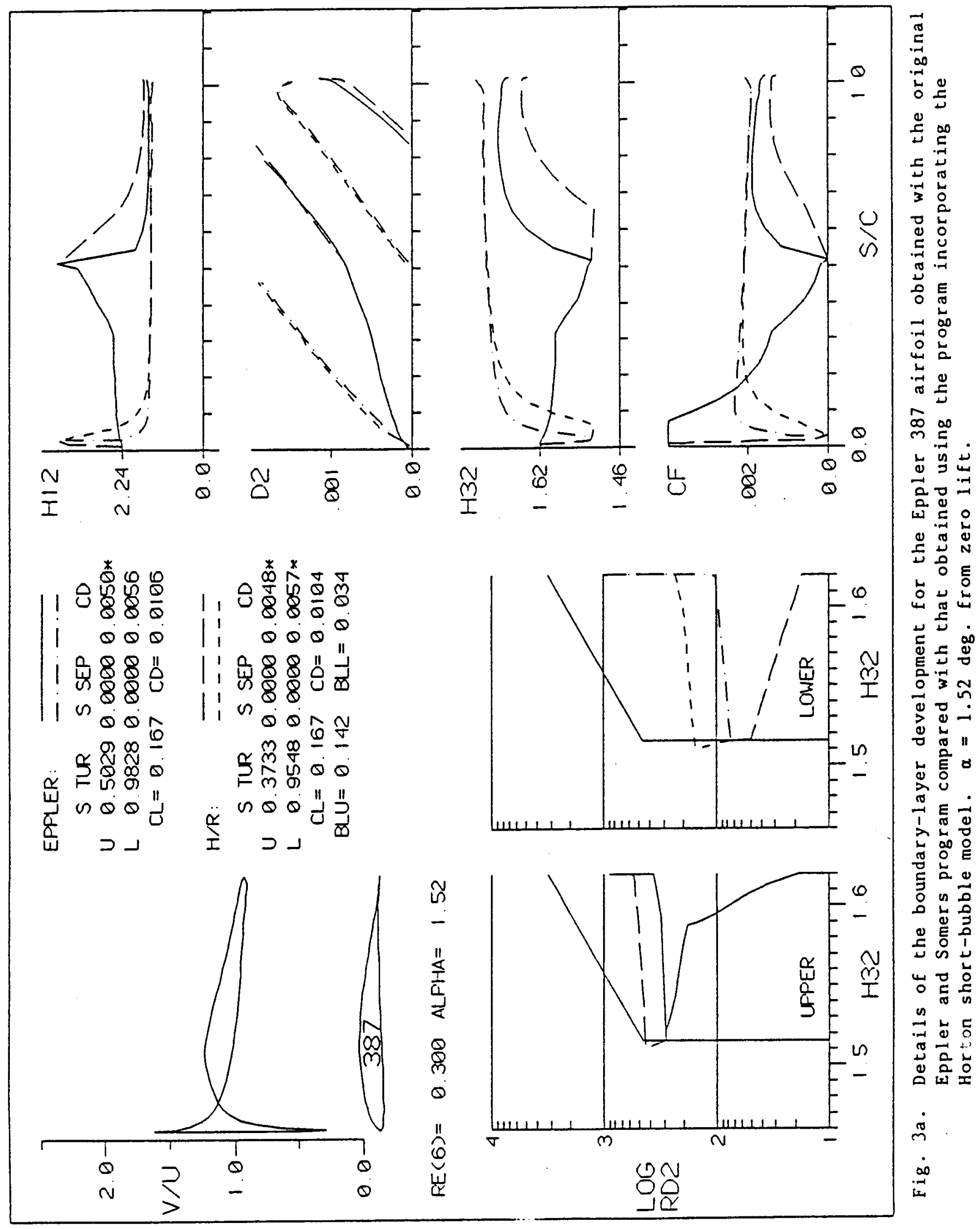




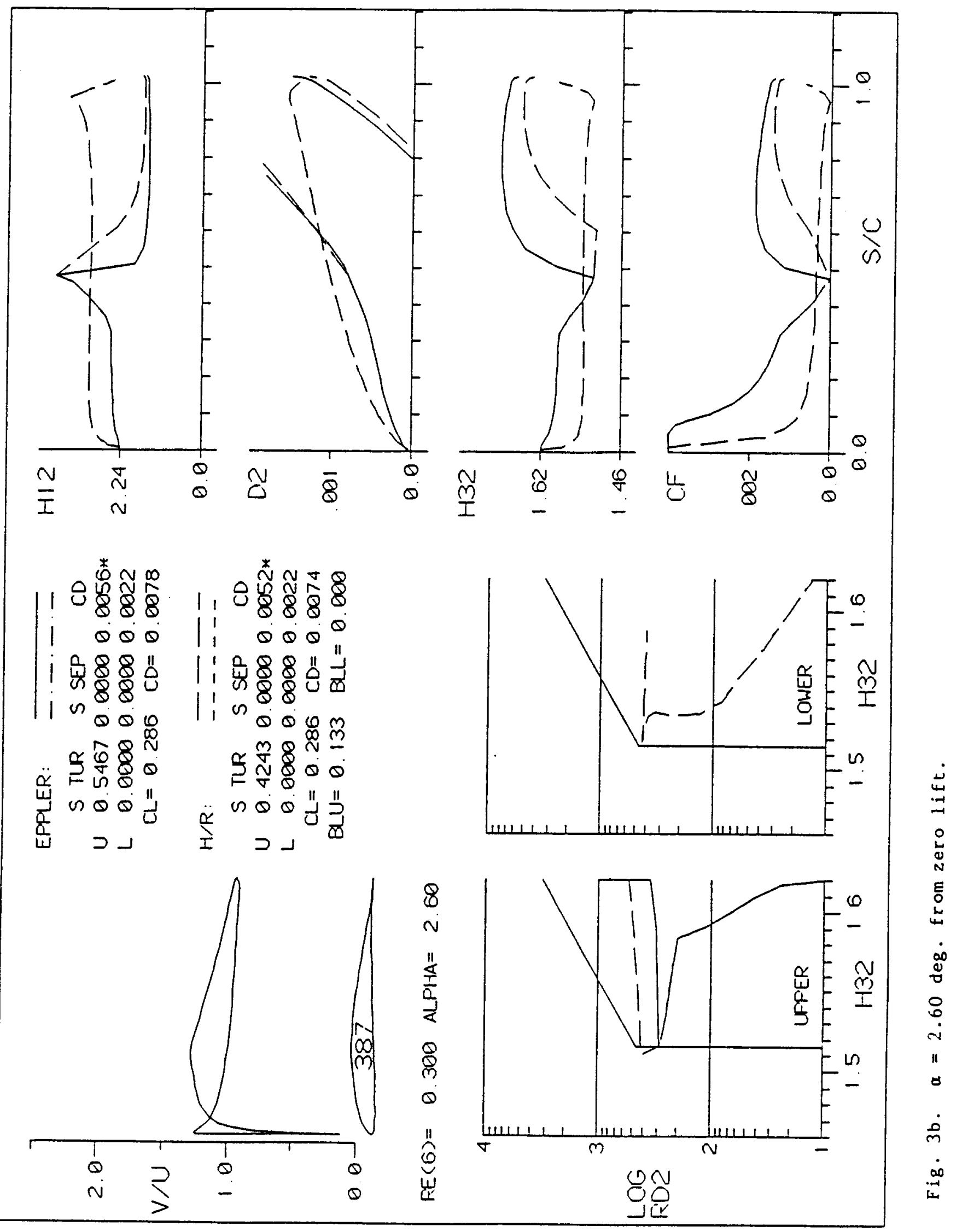




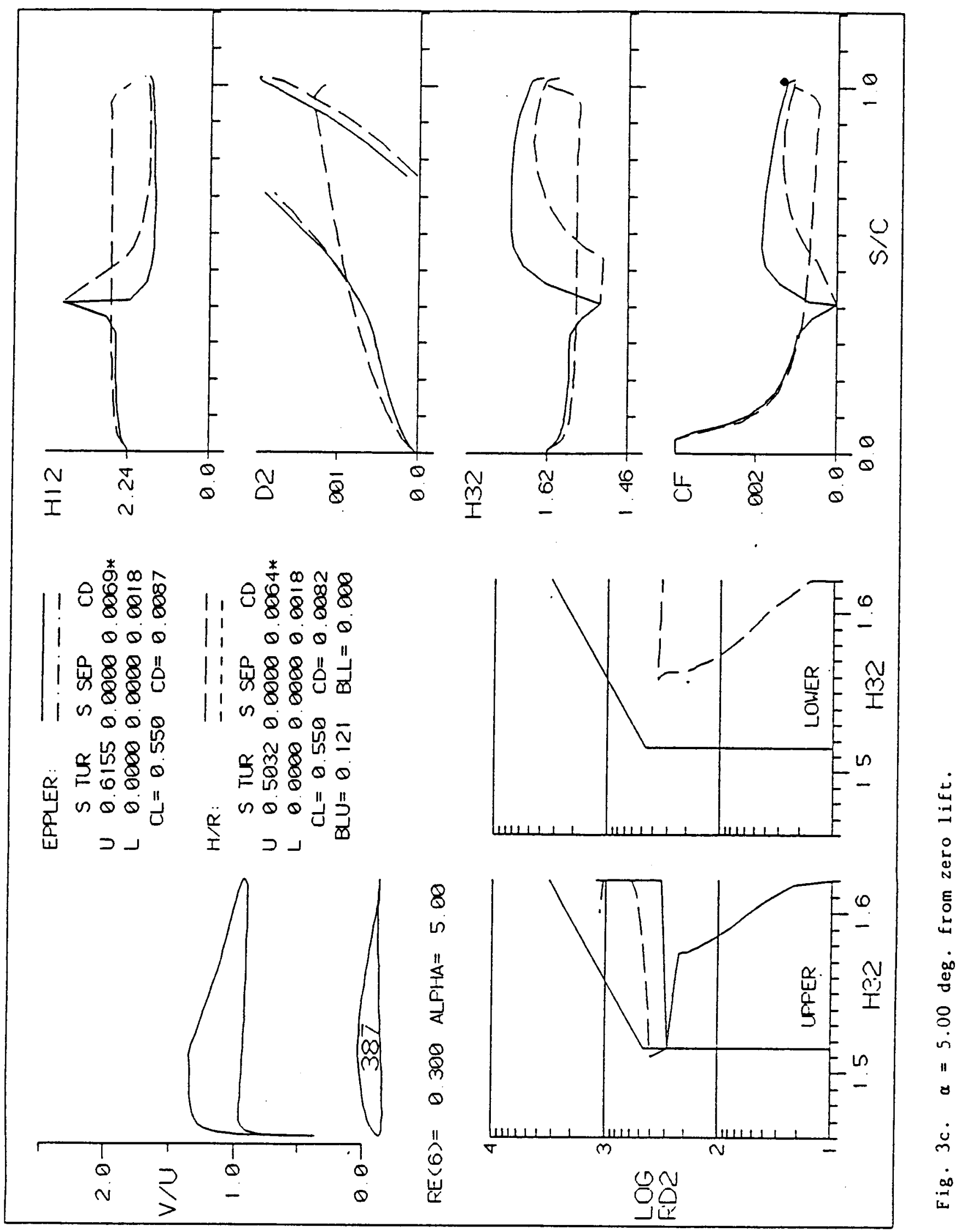




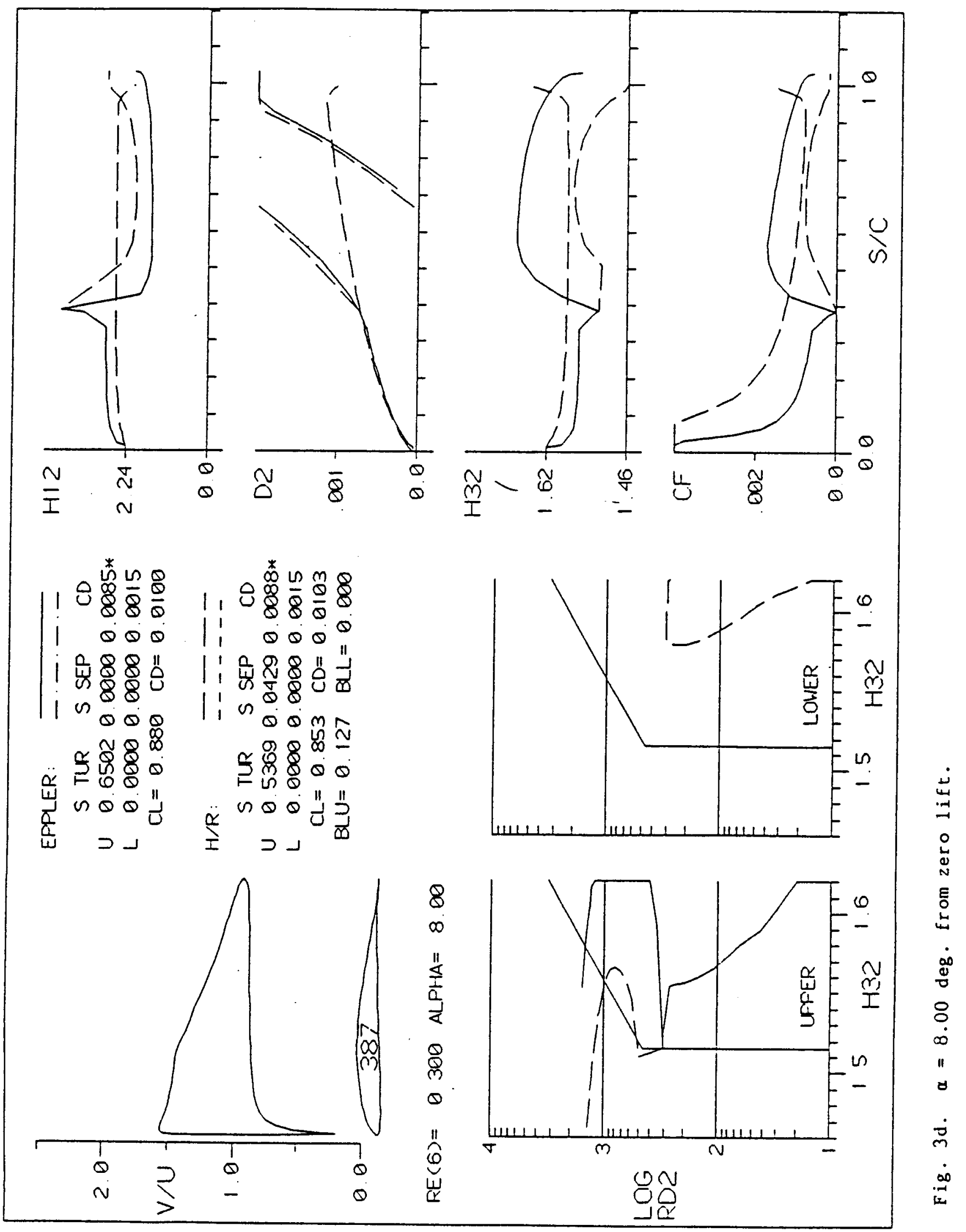




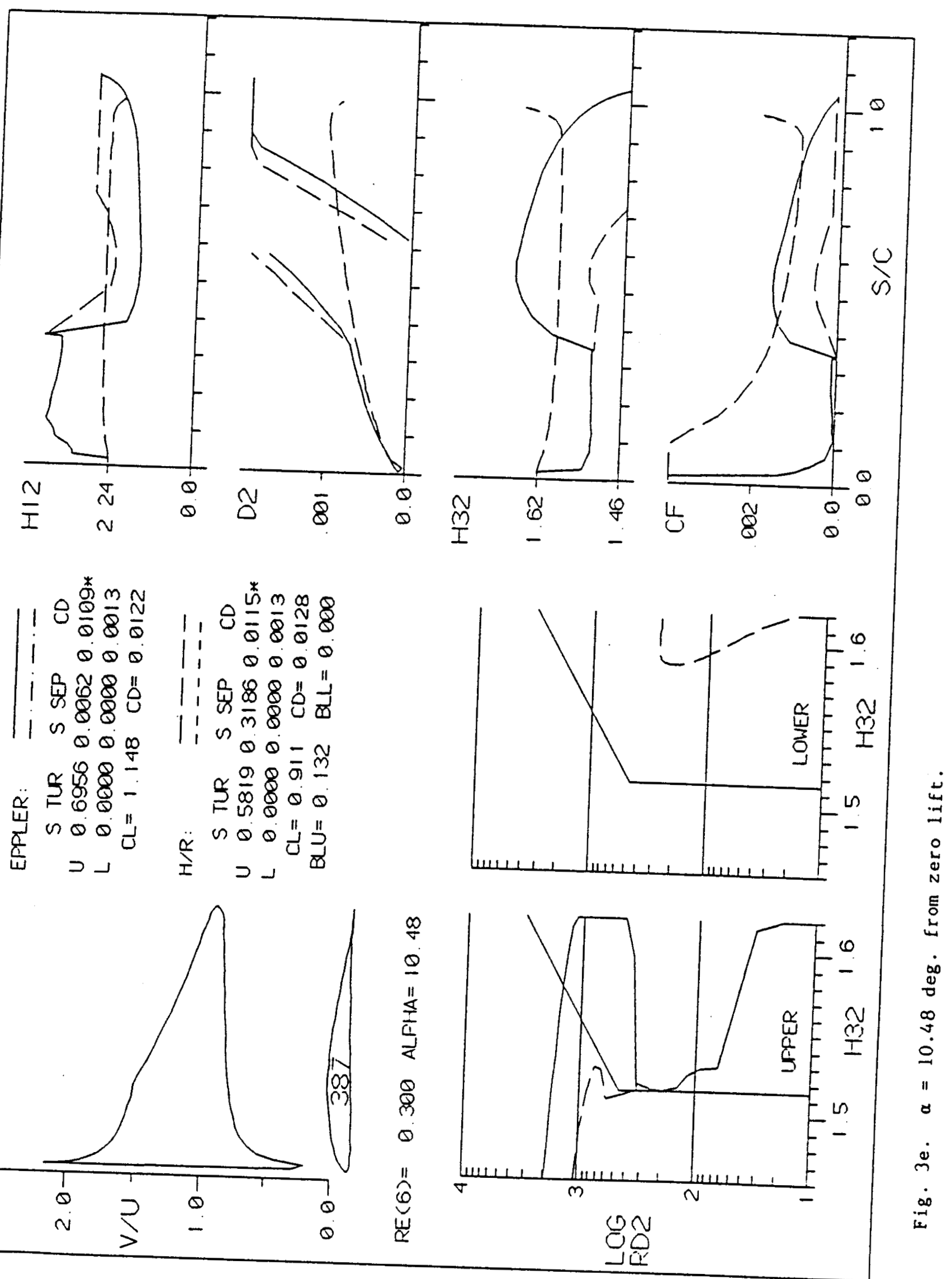




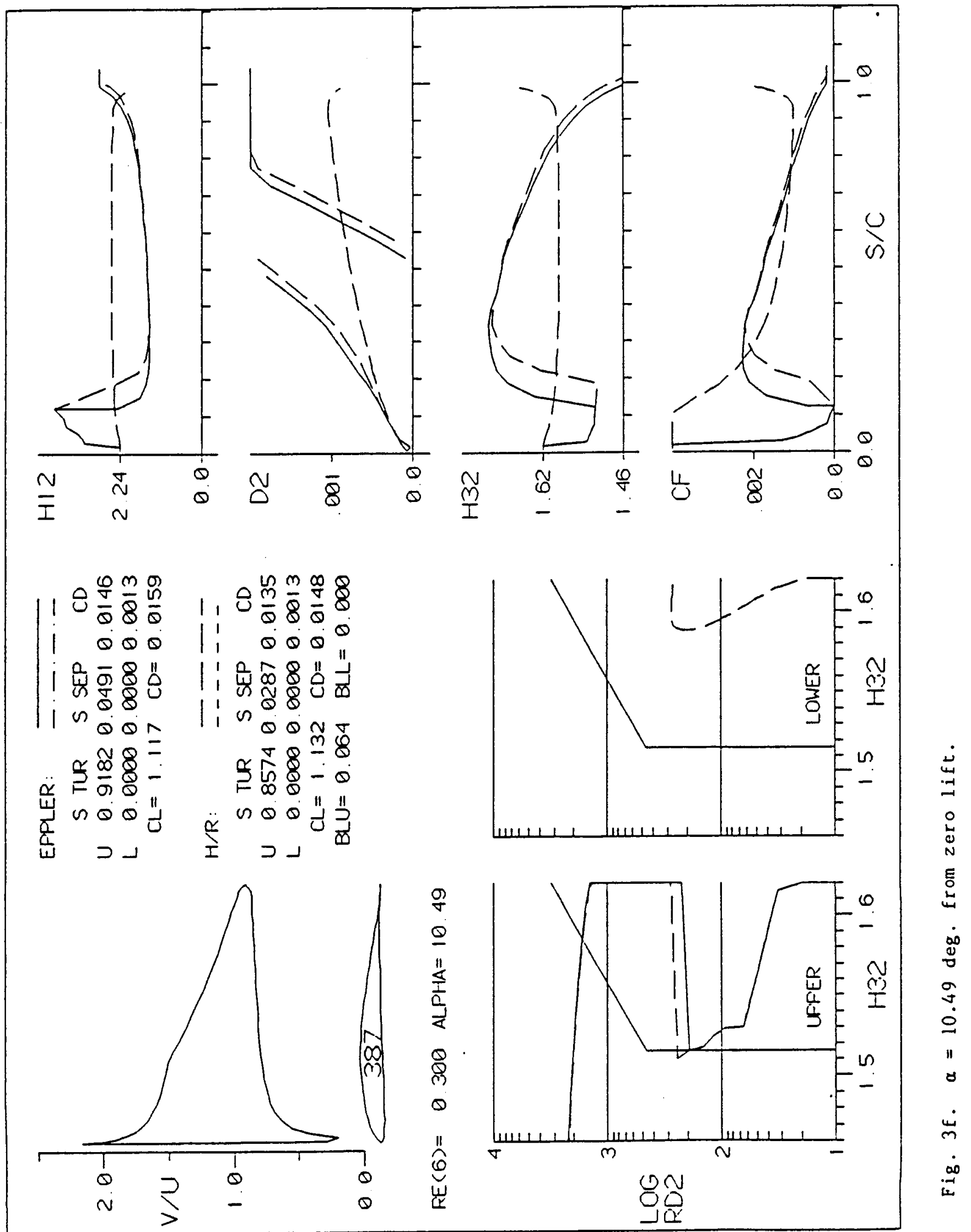

